\title{
Temporal persistence of frustration effects in monkeys and rats'
}

JOHN W. DAVE NPORT, CHARLES F. FLAHERTY AND JAMES P, DYRUD

WISCONSIN REGIONAL PRIMATE RESEARCH CENTER, UNIVERSITY OF WISCONSIN

Rhesus, stumptail, and squirrel monkeys and rats were tested in various retractable-lever analogues of the double runway commonly used to study the Amsel frustration effect in rats. Variation of the midtrial interval (MTI), analogous to midbox confinement time in the runway, showed response speed in the second half of a trial to be an increasing function of the MTI following first-half reward and to persist at a supernormal level on frustration trials, suggesting the operation of two types of frustration effect which persist beyond $40 \mathrm{sec}$.

In a previous report (Davenport \& Thompson, 1965) the Amsel frustration effect (FE) was demonstrated in stumptail monkeys in a dual-retractable-lever analogue of the double runway in which $\mathrm{S}$ completed a fixed number of responses on the left lever followed by an equal number of responses on the right lever. The first half of each trial ended with either reward ( $R$ trial) or nonreward ( $F$ trial), and the left and right halves of a trial were separated by a midtrial interval (MTI) during which both levers were retracted. The FE, defined as faster right-hand responding on $\mathbf{F}$ than on $\mathbf{R}$ trials, appeared consistently in all Ss whenever the MTI was $5 \mathrm{sec}$.

In this report we present data from subsequent research bearing on the temporal persistence of frustration as revealed by experimental manipulation of the MTI. In the course of this work further generality of the FE, to other species and other automated 2-lever procedures, was demonstrated.

\section{Method}

Ss were two rhesus monkeys (Macaca mulatta), two stumptail monkeys (Macaca speciosa), two squirrel monkeys (Saimiri sciurea), and two albino rats. All were adults having previous experience in leverpressing situations involving intermittent reinforcement.

Three Lehigh Valley 1317 monkey test chambers and a Lehigh 1316 rat chamber were used. Each chamber was equipped as in the previous study (Davenport \& Thompson, 1965), with 97-mg sucrose pellets for the macaques, 75-mg Ciba banana pellets for the squirrel monkeys, and 45-mg Noyes rat pellets for the rats. The basic trial procedure was the same as in the previous study except for the MTI and reinforcement schedule manipulations and the use of a longer intertrial interval averaging $2 \mathrm{~min}$.

The Ss received 100 sessions involving a total of 2,080 trials. In the first 84 sessions, the MTI was held constant at $5 \mathrm{sec}$. While the Ss were given baseline training and frustration test sessions on three different 2-lever schedules, designated $\mathrm{DT}-\mathrm{FR} 1_{\mathrm{L}} \mathrm{FR} 1_{\mathrm{R}}$ (single response on each lever), DT-FR1 ${ }_{L}$ DRL $3_{R}$ (single response on the left lever, reward for a single right-lever response having a latency of $3 \mathrm{sec}$. or longer), and $\mathrm{DT}-\mathrm{FR} 1_{\mathrm{L}} \mathrm{DRL} 6_{\mathrm{R}}$ (right-lever latency requirement increased to 6 sec.).

The MTI was manipulated in a series of four 4-day cycles in sessions $85-100$ with the last of these three schedules continued. In each cycle the MTI was varied within Ss among the values of $5,10,20$, and 40 sec. in counterbalanced orders across Ss, with values for a given $\mathrm{S}$ changing from day to day. In each session there were five $F$ trials randomly interspersed among five $\mathbf{R}$ trials, and the assigned MTI value for a given session was the same for both types of trial.

Results

At least one member of each species pair, and usually both members, showed consistent $F E^{\prime}$ 's at MTI $=5 \mathrm{sec}$. under each of the three schedules used. In general the two schedules containing a DRL contingency in the second half of a trial revealed the FE's more clearly in the early sessions of their exposure than did the DT-FR1 $1_{L} F R 1_{R}$ schedule, but after 30 to 40 sessions on the DT-FR1 ${ }_{L}$ DRL6 ${ }_{R}$ schedule, only one member of each pair continued to exhibit the $\mathrm{FE}$.

Results of the MTI variation for these four Ss are shown in Fig. 1. The FE, as usually defined in terms of $\mathrm{R}-\mathrm{F}$ speed differences in right-lever responding, is

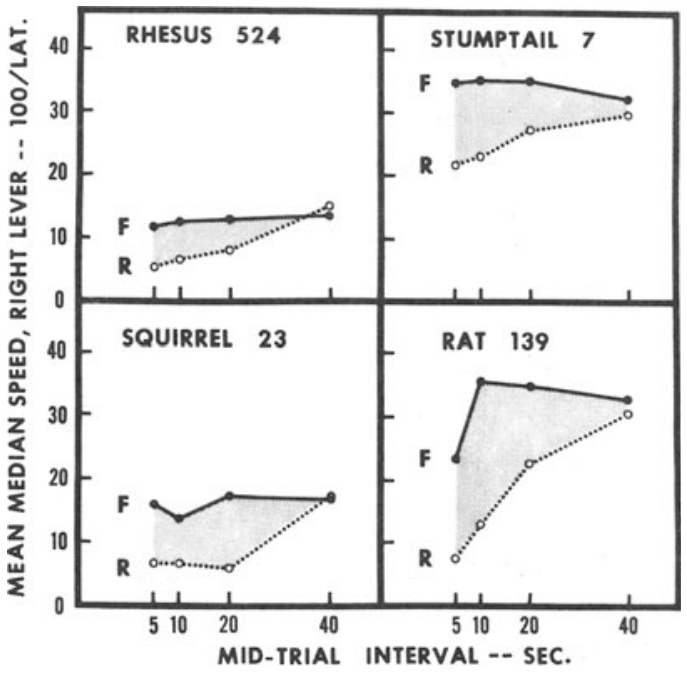

Fig. 1. Right-lever starting speeds for one member of each species pair in sessions 85-100. 
indicated by the shading in these graphs. This shaded area diminished with increasing MTI durations in all four cases but in every instance the diminution was primarily due to an increase in $\mathrm{R}$-trial speed rather than a decline in F-trial speed. In an analysis of variance all main effects and double interactions involving the MTI, R-vs.-F trials, and Ss (species) factors were significant (beyond the .005 level in five cases, beyond the .025 level for Sis-by-MTI); there was no significant triple interaction. A subsequent Newman-Keuls test showed R-trial speed at MTI $=40 \mathrm{sec}$. to be significantly above that of all other MTI levels. The Ss (not included in the analysis) whose FEs disappeared prior to the MTI variation also showed increased $R$-trial speeds with increasing MTI durations and increased F-trial speeds as well.

Supporting these results were the data from a continuation of the previous experiment (Davenport \& Thompson, 1965), in which four stumptail macaques were tested on a DT-FR20 ${ }_{\mathrm{L}}$ FR20 ${ }_{\mathrm{R}}$ schedule. With the same four MTI values manipulated in nine 4-day cycles, three of the four Ss showed MTI functions similar to those in Fig. 1 on the first 10 trials of their 20-trial sessions. Possibly because of the relatively short (30-sec. average) intertrial interval used, however, this pattern of responding disappeared on later session trials.

Discussion

The consistent finding in this research was that increasing the MTI from 5 to 40 sec. produces an elevation of $\mathrm{R}$-trial speed rather than a decline in
F-trial speed. This result was not revealed in the analogous runway studies with rats by Amsel \& Roussel (1952) and Mackinnon \& Amsel (1964), since in those studies midbox confinement time was varied on $\mathrm{F}$ trials only. Our interpretation of the finding is that frustration due to nonreward is probably not completely dissipated after 40 sec. of midtrial waiting and that a second type of frustration effect, which may be identified with that apparently operating in the midboxdelay study by Holder et al (1957), is generated by delaying the onset of the second half of an $R$ trial. Since this second frustration effect may also operate on $F$ trials and both types of frustration may be expected to dissipate eventually, future attempts to map the temporal course of frustration effects will require a wider range of MTI values and more analytical procedures for separating the sources of frustration.

\section{References}

Amsel, A., \& Roussel, J. Motivational properties of frustration: I. Effect on a running response of the addition of frustration to the motivational complex. J. exp. Psychol., 1952, 43, 363-368.

Davenport, J. W., \& Thompson, C. I. The Amsel frustration effect in monkeys. Psychon. Sci., 1965, 3, 481-482.

Holder, W. B., Marx, M. H., Holder, E. E., \& Collier, G. Response strength as a function of delay of reward in a runway. J. exp. Psychol., 1957, 53, 316-323.

Mackinnon, J. R., \& Amsel, A. Magnitude of the frustration effect as a function of confinement and detention in the frustrating situation. J. exp. Psychol., 1964, 67, 468-474.

\section{Note}

1. This research was supported by grant FR-00167 from the National Institutes of Health. 